\title{
6D attractors and black hole microstates
}

\author{
Seyed Morteza Hosseini, ${ }^{a}$ Kiril Hristov, ${ }^{b}$ Achilleas Passias ${ }^{c}$ and Alberto Zaffaroni ${ }^{d, e}$ \\ ${ }^{a}$ Kavli IPMU (WPI), UTIAS, The University of Tokyo, Kashiwa, \\ Chiba 277-8583, Japan \\ ${ }^{b}$ Institute for Nuclear Research and Nuclear Energy, Bulgarian Academy of Sciences, \\ Tsarigradsko Chaussee 72, 1784 Sofia, Bulgaria \\ ${ }^{c}$ Department of Physics and Astronomy, Uppsala University, \\ Box 516, SE-751 20 Uppsala, Sweden \\ ${ }^{d}$ Dipartimento di Fisica, Università di Milano-Bicocca, \\ I-20126 Milano, Italy \\ ${ }^{e}$ INFN - Sezione di Milano-Bicocca, \\ I-20126 Milano, Italy \\ E-mail: morteza.hosseini@ipmu.jp, khristov@inrne.bas.bg, \\ achilleas.passias@physics.uu.se, alberto.zaffaroni@mib.infn.it
}

ABSTRACT: We find a family of $\mathrm{AdS}_{2} \times \mathcal{M}_{4}$ supersymmetric solutions of the six-dimensional $\mathrm{F}(4)$ gauged supergravity coupled to one vector multiplet that arises as a low energy description of massive type IIA supergravity on (warped) $\mathrm{AdS}_{6} \times S^{4} . \mathcal{M}_{4}$ is either a KählerEinstein manifold or a product of two Riemann surfaces with a constant curvature metric. These solutions correspond to the near-horizon region of a family of static magnetically charged black holes. In the case where $\mathcal{M}_{4}$ is a product of Riemann surfaces, we successfully compare their entropy to a microscopic counting based on the recently computed topologically twisted index of the five-dimensional $\mathcal{N}=1 \mathrm{USp}(2 N)$ theory with $N_{f}$ fundamental flavors and an antisymmetric matter field. Furthermore, our results suggest that the near-horizon regions exhibit an attractor mechanism for the scalars in the matter coupled $\mathrm{F}(4)$ gauged supergravity, and we give a proposal for it.

KeYwords: Black Holes in String Theory, AdS-CFT Correspondence, Supergravity Models

ArXiv EPrint: 1809.10685 


\section{Contents}

1 Introduction 1

2 Matter coupled F(4) gauged supergravity 4

$\begin{array}{lll}3 & \text { The } \mathrm{AdS}_{6} \text { vacuum } & 7\end{array}$

4 The $\mathrm{AdS}_{4} \times \Sigma_{\mathfrak{g}}$ solution $\quad 9$

5 The $\mathrm{AdS}_{2} \times \Sigma_{\mathfrak{g}_{1}} \times \Sigma_{\mathfrak{g}_{2}}$ solution 11

6 The $\mathrm{AdS}_{2} \times \mathcal{M}_{4}$ solution 13

$\begin{array}{ll}\text { A Conventions } & 15\end{array}$

\section{Introduction}

Recently there has been some progress in understanding the microscopic origin of the Bekenstein-Hawking entropy of supersymmetric asymptotically anti-de Sitter (AdS) black holes. In particular, the microscopic entropy of certain four-dimensional static, dyonic, BPS black holes [1-5], which can be embedded in $\mathrm{AdS}_{4} \times S^{7}$, has been reproduced by a field theory calculation based on the topologically twisted index [6] of the dual ABJM theory in the large $N$ limit [7-9]. These black holes have an $\mathrm{AdS}_{2} \times \Sigma_{\mathfrak{g}}$ near-horizon geometry, where $\Sigma_{\mathfrak{g}}$ is a Riemann surface of genus $\mathfrak{g}$. The topologically twisted index is the partition function of the dual field theory on $\Sigma_{\mathfrak{g}} \times S^{1}$, partially topologically $A$-twisted along $\Sigma_{\mathfrak{g}}$. Specifically, the index $Z\left(p_{I}, \Delta_{I}\right)$ is a function of a set of magnetic charges $p_{I}$ and complexified chemical potentials $\Delta_{I}$ for the global symmetries of the theory. The statistical entropy $S_{\mathrm{BH}}$ of the black holes with purely magnetic charges is then obtained by evaluating $Z\left(p_{I}, \Delta_{I}\right)$ at its critical point $\bar{\Delta}_{I}$ :

$$
\mathcal{I}_{\mathrm{SCFT}}\left(p_{I}, \bar{\Delta}_{I}\right) \equiv \log Z\left(p_{I}, \bar{\Delta}_{I}\right)=S_{\mathrm{BH}}\left(p_{I}\right) .
$$

This procedure was dubbed $\mathcal{I}$-extremization in [7]. These results have been generalized to different black holes in four dimensions and black strings in five dimensions [10-21]. For other interesting progresses in this context see [22-31].

The five-dimensional topologically twisted index, which is the partition function of a five-dimensional $\mathcal{N}=1$ theory on $\mathcal{M}_{4} \times S^{1}$ with an Abelian topological twist along $\mathcal{M}_{4}$, has been recently computed when $\mathcal{M}_{4}$ is a toric Kähler manifold [32] or the product of two Riemann surfaces [32, 33]. Therein, the $\mathcal{N}=1 \mathrm{USp}(2 N)$ gauge theory with $N_{f}$ hypermultiplets in the fundamental representation and one hypermultiplet in the antisymmetric 
representation of $\operatorname{USp}(2 N)$, arising on the worldvolume of D4-branes near D8-branes and orientifolds [34], has been analyzed in the large $N$ limit. With some assumptions on the relevant saddle-point, the large $N$ limit of the index for $\mathcal{M}_{4}=\Sigma_{\mathfrak{g}_{1}} \times \Sigma_{\mathfrak{g}_{2}}$ has been evaluated as a function of magnetic charges and chemical potentials for the Cartan subgroup of the $\mathrm{SU}(2)_{M}$ global symmetry of the theory [32]. ${ }^{1}$ This result provides a prediction for the entropy of a family of $\mathrm{AdS}_{6}$ magnetically charged black holes in massive type IIA supergravity. This prediction has been successfully tested for the only existing black hole solution, the so-called universal one $[35,36]$ with a particular value of the magnetic charge, using the results for the entropy given in [37]. It is the purpose of this paper to find new black hole solutions, explicitly depending on a set of magnetic charges, and show that their entropy is correctly accounted by the topologically twisted index.

To this end, we will consider a six-dimensional truncation of the supersymmetric warped $\mathrm{AdS}_{6} \times S^{4}$ background of massive type IIA supergravity [38] dual to the USp $(2 N)$ theory. This truncation is described by an $\mathrm{F}(4)$ gauged supergravity coupled to vector multiplets [39-42]. Furthermore, we will restrict ourselves to one vector multiplet corresponding to the Cartan subgroup of the $\mathrm{SU}(2)_{M}$ global symmetry of the five-dimensional superconformal field theory (SCFT).

As a warm-up, and to test the consistency of the truncation, we consider the background $\mathrm{AdS}_{4} \times \Sigma_{\mathfrak{g}}$ which corresponds to a twisted compactification of the five-dimensional field theory on $\Sigma_{\mathfrak{g}}$. We successfully compare the free energy of the solution with the field theory computation in [33] and the ten-dimensional gravity computation in [43].

We then find new black hole horizon geometries of the form $\mathrm{AdS}_{2} \times \Sigma_{\mathfrak{g}_{1}} \times \Sigma_{\mathfrak{g}_{2}}$. We turn on an Abelian gauge field inside the SU(2) R-symmetry that performs the topological twist by cancelling the spin connection, and two magnetic fluxes $p_{1}$ and $p_{2}$ (one along each Riemann surface) for the U(1) gauge field in the additional vector multiplet. We thus have a two-parameter family of magnetically charged black holes. We compare the entropy with the value of the topologically twisted index $Z\left(p_{1}, p_{2}, \Delta\right)$ that also depends on a chemical potential for the $\mathrm{U}(1) \subset \mathrm{SU}(2)_{M}$ symmetry and we find that the statistical entropy $S_{\mathrm{BH}}$ of the black holes as a function of the magnetic charges is obtained by evaluating $Z\left(p_{1}, p_{2}, \Delta\right)$ at its critical point $\bar{\Delta}$ :

$$
\mathcal{I}_{\mathrm{SCFT}}\left(p_{1}, p_{2}, \bar{\Delta}\right) \equiv \log Z\left(p_{1}, p_{2}, \bar{\Delta}\right)=S_{\mathrm{BH}}\left(p_{1}, p_{2}\right) .
$$

With a convenient democratic parameterization ${ }^{2}$ for the fluxes and chemical potentials the explicit form of the index can be written as [32]

$$
\mathcal{I}_{\mathrm{SCFT}}\left(\mathfrak{s}_{I}, \mathfrak{t}_{I}, \Delta_{I}\right)=\frac{4 \sqrt{2}}{15} \frac{N^{5 / 2}}{\sqrt{8-N_{f}}} \sum_{I, J=1}^{2} \mathfrak{s}_{I} \mathfrak{t}_{J} \frac{\partial^{2}\left(\Delta_{1} \Delta_{2}\right)^{3 / 2}}{\partial \Delta_{I} \partial \Delta_{J}} .
$$

\footnotetext{
${ }^{1} \mathrm{~A}$ different proposal was discussed in [33]. In this paper we use the proposal in [32] that nicely matches the entropy of the black holes.

${ }^{2}$ The $\Delta_{I}, I=1,2$, parameterize the Cartan of the $\mathrm{SU}(2)_{R}$ and the $\mathrm{SU}(2)_{M}$ symmetry of the $\mathrm{USp}(2 N)$ theory. They satisfy the constraint (5.11). Similarly, one can introduce a redundant, but democratic, parameterization for the fluxes as in (5.7). With such a choice, the topologically twisted index is a homogeneous function of $\Delta_{I}, \mathfrak{s}_{I}$ and $\mathfrak{t}_{I}$. See [32] for details.
} 
This structure is reminiscent of an analogous result for $\mathrm{AdS}_{4}$ black holes $[7-10,17,18]$. This analogy and the relation to other interesting field theory quantities like the $S^{5}$ free energy and the effective twisted superpotential of the partial compactification on one of the Riemann surfaces, were discussed in detail in [32].

We also obtain $\mathrm{AdS}_{2} \times \mathcal{M}_{4}$ horizon geometries where $\mathcal{M}_{4}$ is a four-dimensional KählerEinstein manifold depending on a magnetic flux along $\mathcal{M}_{4}$. We find a simple and intriguing expression for the entropy suggesting that the computation in [32] could be generalized to this case too. We leave this for future work.

In gravity, the field theory chemical potential $\Delta$ can be associated with the horizon value of the vector multiplet scalar field $\phi_{3}$. With a convenient parameterization, we find that the functional $\mathcal{I}_{\mathrm{SCFT}}\left(p_{1}, p_{2}, \bar{\Delta}\right)$ coincides with the area of the horizon divided by $4 G_{\mathrm{N}}$, where $G_{\mathrm{N}}$ is the six-dimensional Newton's constant, as a function of $\phi_{3}$. This is the attractor mechanism in six-dimensional gauged supergravity: after expressing all the fields in the gravity multiplet in terms of vector multiplet scalars using the BPS equations, the remaining BPS equations are equivalent to the extremization of the area of the horizon as a functional of vector multiplet scalars, and the critical value of this functional is the entropy. We see that the $\mathcal{I}$-extremization principle is equivalent to the attractor mechanism in six-dimensional gauged supergravity, ${ }^{3}$ thus generalizing what was found for $\mathrm{AdS}_{4}$ black holes in $[7-9,17,18]$.

More explicitly, we find that a central role is played by the quantity (3.4)

$$
\mathcal{I}_{\mathrm{AdS}_{6}}\left(X^{I}\right)=-\frac{1}{3 \pi G_{\mathrm{N}}}\left(X^{1} X^{2}\right)^{3 / 2},
$$

where $X^{I}\left(\phi_{3}\right)(I=1,2)$, defined in (2.12), are the gravity counterpart of $\Delta_{I}$ in (1.3). This six-dimensional quantity is reminiscent and can be thought of as the analogue of the prepotential $\mathcal{F}_{\text {sugra }}\left(X^{I}\right)$ in four-dimensional $\mathcal{N}=2$ gauged supergravity. Indeed, we will find that the attractor equations for $\mathrm{AdS}_{4}$ vacua correspond to extremizing

$$
\mathcal{I}_{\mathrm{AdS}_{4}}\left(X^{I}\right)=\frac{8 \pi}{27} \sum_{I=1}^{2} \mathfrak{s}_{I} \frac{\partial \mathcal{I}_{\mathrm{AdS}_{6}}\left(X^{I}\right)}{\partial X^{I}},
$$

and attractor equations for black holes correspond to extremizing

$$
\mathcal{I}_{\mathrm{AdS}_{2}}\left(X^{I}\right)=-\frac{\operatorname{vol}\left(\mathcal{M}_{4}\right)}{108} \sum_{I, J=1}^{2} \mathfrak{s}_{I} \mathfrak{s}_{J} \frac{\partial^{2} \mathcal{I}_{\mathrm{AdS}_{6}}\left(X^{I}\right)}{\partial X^{I} \partial X^{J}},
$$

for $\mathcal{M}_{4}$ being a Kähler-Einstein manifold, and to extremizing

$$
\mathcal{I}_{\mathrm{AdS}_{2}}\left(X^{I}\right)=-\frac{4 \pi^{2}}{27} \sum_{I, J=1}^{2} \mathfrak{s}_{I} \mathfrak{t}_{J} \frac{\partial^{2} \mathcal{I}_{\mathrm{AdS}_{6}}\left(X^{I}\right)}{\partial X^{I} \partial X^{J}},
$$

for $\mathcal{M}_{4}=\Sigma_{\mathfrak{g}_{1}} \times \Sigma_{\mathfrak{g}_{2}}$. Here $\mathfrak{s}_{I}$ and $\mathfrak{t}_{I}$ are the magnetic charges — see (4.6), (5.7) and (6.6). This is similar to the attractor mechanism in four-dimensional $\mathcal{N}=2$ gauged supergravity $[1,2]$. We thus expect that, in more general $\mathrm{F}(4)$ gauged supergravites coupled to vector

\footnotetext{
${ }^{3}$ For the attractor mechanism in six-dimensional ungauged supergravity, instead, see e.g. [44, 45] and references therein.
} 
multiplets, the attractor equations for AdS solutions supported by magnetic fluxes are given by extremizing expressions of the form (1.4)-(1.7) with a suitable function $\mathcal{I}_{\mathrm{AdS}_{6}}\left(X^{I}\right)$, homogeneous of degree three.

The structure of this paper is as follows. In section 2 we discuss general aspects of the $\mathrm{F}(4)$ gauged supergravity coupled to vector multiplets. In section 3 we discuss the $\mathrm{AdS}_{6}$ vacuum and an interesting partially off-shell version of its free energy that we relate to its field theory counterpart. In section 4 we consider the background $\operatorname{AdS}_{4} \times \Sigma_{\mathfrak{g}}$ with a topological twist on $\Sigma_{\mathfrak{g}}$ and we successfully compare the free energy of the solution with the field theory computation in [33] and the ten-dimensional gravity computation in [43]. In section 5 we obtain a two-parameter family of black hole horizons $\mathrm{AdS}_{2} \times$ $\Sigma_{\mathfrak{g}_{1}} \times \Sigma_{\mathfrak{g}_{2}}$ and successfully reproduce their entropy using the topologically twisted index. In section 6 we find a one-parameter family of black hole horizons $\mathrm{AdS}_{2} \times \mathcal{M}_{4}$ where $\mathcal{M}_{4}$ is a four-dimensional Kähler-Einstein manifold. Our conventions and some useful formulae are collected in appendix A.

Note added: while we were writing this work, we became aware of [46] which has some overlaps with the results presented here.

\section{Matter coupled F(4) gauged supergravity}

We consider a six-dimensional truncation of the supersymmetric warped $\operatorname{AdS}_{6} \times S^{4}$ background of massive type IIA supergravity [38] described by an F(4) gauged supergravity coupled to vector multiplets. The minimal $\mathrm{F}(4)$ gauged supergravity was written in [40] and coupled to matter in $[41,42] . \mathrm{F}(4)$ is the relevant superalgebra for five-dimensional superconformal field theories and its bosonic subalgebra is $S \mathrm{O}(5,2) \times \mathrm{SU}(2)_{R}$.

The bosonic part of the six-dimensional gravity multiplet consists of the metric $g_{\mu \nu}$, four vectors $A^{\alpha}, \alpha=0,1,2,3$, a two-form $B_{\mu \nu}$ and the dilaton $\sigma$. It is useful to split $\alpha=$ $(0, r)$ where $r=1,2,3$ is an index in the adjoint representation of $\mathrm{SU}(2)_{R}$. The fermionic components are a gravitino $\psi_{\mu}^{A}$ and a spin one-half fermion $\chi^{A}, A=1,2$, transforming in the fundamental representation of $\mathrm{SU}(2)_{R}$.

The vector multiplet in six-dimensions contains a gauge field $A_{\mu}$, four scalars $\phi_{\alpha}$ and a spin one-half fermion $\lambda_{A}$. With $n_{\mathrm{V}}$ vector multiplets, the $4 n_{\mathrm{V}}$ scalar fields parameterize the coset space

$$
\frac{\mathrm{SO}\left(4, n_{\mathrm{V}}\right)}{\mathrm{SO}(4) \times \mathrm{SO}\left(n_{\mathrm{V}}\right)}
$$

It is convenient to encode the scalar fields into a coset representative $L_{\Sigma}^{\Lambda} \in \operatorname{SO}\left(4, n_{\mathrm{V}}\right)$, where indices are split as follows $\Lambda=(\alpha, I)$ with $I=1, \ldots n_{\mathrm{V}}$. A subgroup $\mathrm{SU}(2)_{R} \times G$ of dimension $3+n_{\mathrm{V}}$ of $\mathrm{SO}\left(4, n_{\mathrm{V}}\right)$ can be gauged.

The bosonic Lagrangian reads $[42]^{4}$

$$
\begin{aligned}
\mathcal{L}= & -\frac{1}{4} R-\frac{1}{8} e^{-2 \sigma} \mathcal{N}_{\Lambda \Sigma} \hat{F}_{\mu \nu}^{\Lambda} \hat{F}^{\Sigma \mu \nu}+\frac{3}{64} e^{4 \sigma} H_{\mu \nu \rho} H^{\mu \nu \rho}+\partial^{\mu} \sigma \partial_{\mu} \sigma-\frac{1}{4} P^{I \alpha \mu} P_{I \alpha \mu}-V \\
& -\frac{1}{64} \epsilon^{\mu \nu \rho \sigma \lambda \tau} B_{\mu \nu}\left(\eta_{\Lambda \Sigma} \hat{F}_{\rho \sigma}^{\Lambda} \hat{F}_{\lambda \tau}^{\Sigma}+m B_{\rho \sigma} \hat{F}_{\lambda \tau}^{0}+\frac{1}{3} m^{2} B_{\rho \sigma} B_{\lambda \tau}\right)
\end{aligned}
$$

\footnotetext{
${ }^{4}$ We follow the conventions of [42]. Notice that [42] employs the unusual convention $F=F_{\mu \nu} \mathrm{d} x^{\mu} \wedge \mathrm{d} x^{\nu}$ for the components of a form. In particular, for them $F_{\mu \nu}=\frac{1}{2}\left(\partial_{\mu} A_{\nu}-\partial_{\nu} A_{\mu}\right)$.
} 
where

$$
\begin{aligned}
\hat{F}_{\rho \sigma}^{\Lambda} & =F_{\rho \sigma}^{\Lambda}-m \delta^{\Lambda 0} B_{\mu \nu} \\
\mathcal{N}_{\Lambda \Sigma} & =L_{\Lambda}{ }^{\alpha}\left(L^{-1}\right)_{\alpha \Sigma}-L_{\Lambda}{ }^{I}\left(L^{-1}\right)_{I \Sigma}, \\
P_{\alpha}^{I} & =\left(L^{-1}\right)^{I}{ }_{\Lambda}\left(\mathrm{d} L^{\Lambda}{ }_{\alpha}-f_{\Gamma}{ }_{\Gamma}{ }_{\Pi} A^{\Gamma} L^{\Pi}{ }_{\alpha}\right),
\end{aligned}
$$

with $f_{\Pi \Gamma}^{\Lambda}$ the structure constants of the gauge group $\mathrm{SU}(2)_{R} \times G$. Here, $g$ is the gauge coupling constant and $m$ is the mass parameter of the massive type IIA supergravity [47].

The supersymmetry variations of the fermions are given by

$$
\begin{aligned}
\delta \psi_{A \mu}= & \nabla_{\mu} \epsilon_{A}-\frac{i}{2} g \sigma_{A B}^{r} A_{r \mu} \epsilon^{B}+\frac{1}{16} e^{-\sigma}\left[\hat{T}_{[A B] \nu \lambda} \gamma_{7}-T_{(A B) \nu \lambda}\right]\left(\gamma_{\mu}{ }^{\nu \lambda}-6 \delta_{\mu}^{\nu} \gamma^{\lambda}\right) \epsilon^{B} \\
& +\frac{i}{32} e^{2 \sigma} H_{\nu \lambda \rho} \gamma_{7}\left(\gamma_{\mu}{ }^{\nu \lambda \rho}-3 \delta_{\mu}^{\nu} \gamma^{\lambda \rho}\right) \epsilon_{A}+S_{A B} \gamma_{\mu} \epsilon^{B} \\
\delta \chi_{A}= & \frac{i}{2} \gamma^{\mu} \partial_{\mu} \sigma \epsilon_{A}+\frac{i}{16} e^{-\sigma}\left[\hat{T}_{[A B] \nu \lambda} \gamma_{7}+T_{(A B) \nu \lambda}\right] \gamma^{\nu \lambda} \epsilon^{B} \\
& +\frac{1}{32} e^{2 \sigma} H_{\nu \lambda \rho} \gamma_{7} \gamma^{\nu \lambda \rho} \epsilon_{A}+N_{A B} \epsilon^{B} \\
\delta \lambda_{A}^{I}= & i P_{r \mu}^{I} \sigma_{A B}^{r} \gamma^{\mu} \epsilon^{B}-i P_{0 \mu}^{I} \epsilon_{A B} \gamma^{7} \gamma^{\mu} \epsilon^{B}+\frac{i}{2} e^{-\sigma} T_{\mu \nu}^{I} \gamma^{\mu \nu} \epsilon_{A}+M_{A B}^{I} \epsilon^{B}
\end{aligned}
$$

where we suppressed the quadratic terms in fermions, $\sigma^{r A}{ }_{B}$ are the Pauli matrices, and we have defined

$$
\hat{T}_{[A B] \nu \lambda}=\epsilon_{A B} L_{0 \Lambda}^{-1} \hat{F}_{\nu \lambda}^{\Lambda}, \quad T_{(A B) \nu \lambda}=\sigma_{A B}^{r} L_{r \Lambda}^{-1} F_{\nu \lambda}^{\Lambda}, \quad T_{I \nu \lambda}=L_{I \Lambda}^{-1} F_{\nu \lambda}^{\Lambda}
$$

In all the above formulae the indices $\Lambda, \Pi, \Gamma, \ldots$ are raised and lowered with the $\mathrm{SO}\left(4, n_{\mathrm{V}}\right)$ invariant metric $\eta_{\Lambda \Sigma}=\operatorname{diag}\{1,1,1,1,-1, \ldots,-1\}$ and the indices $A, B, \ldots$ with the $\mathrm{SU}(2)_{R}$ tensor $\epsilon_{A B}$. We refer to the appendix for conventions, for the explicit form of the potential $V$, and the fermion mass matrices $S_{A B}, N_{A B}, M_{A B}^{I}$ appearing in (2.4).

The five-dimensional superconformal field theory dual to the warped background $\mathrm{AdS}_{6} \times S^{4}$ has a gauge group $\operatorname{USp}(2 N), N_{f}$ hypermultiplets in the fundamental representation and one hypermultiplet in the antisymmetric representation. The theory has an $\mathrm{SU}(2)_{R} \times \mathrm{SU}(2)_{M} \times \mathrm{SO}\left(2 N_{f}\right) \times \mathrm{U}(1)_{I}$ symmetry [34]. ${ }^{5}$ The global $\mathrm{SU}(2)_{M}$ acts on the antisymmetric field, $\mathrm{SO}\left(2 N_{f}\right)$ on the fundamentals and $\mathrm{U}(1)_{I}$ is the conserved instanton current.

We thus just consider a supergravity containing one vector multiplet, $n_{\mathrm{V}}=1$, corresponding to the $\mathrm{U}(1)$ subgroup of the global $\mathrm{SU}(2)_{M}$. We will consistently set to zero all gauge fields except $A_{\mu}^{r=3}$ in $\mathrm{SU}(2)_{R}$ and $A_{\mu}^{I=1}$ that are needed for the twisting and to provide magnetic charges for the black holes. We will also require the scalar fields in the vector multiplet $\phi_{\alpha}, \alpha=0,1,2,3$, to be neutral under $A_{\mu}^{r=3}$ and this restricts the nonzero components to $\phi_{0}$ and $\phi_{3}$. For purely magnetic black holes we can find solutions with $\phi^{0}=0$ and we further restrict to this case. ${ }^{6}$ A convenient parameterization of the scalar

\footnotetext{
${ }^{5}$ This is non-perturbatively enhanced to $\mathrm{SU}(2)_{R} \times \mathrm{SU}(2)_{M} \times \mathrm{E}_{N_{f}+1}$.

${ }^{6}$ One can think of the $\phi_{0}=0$ as analogous to the vanishing of the axions for the $\mathrm{AdS}_{4}$ magnetic black holes [1-3].
} 
coset is given by [48-50]

$$
L_{\Sigma}^{\Lambda}=\left(\begin{array}{ccccc}
1 & 0 & 0 & 0 & 0 \\
0 & 1 & 0 & 0 & 0 \\
0 & 0 & 1 & 0 & 0 \\
0 & 0 & 0 & \cosh \left(\phi_{3}\right) & \sinh \left(\phi_{3}\right) \\
0 & 0 & 0 & \sinh \left(\phi_{3}\right) & \cosh \left(\phi_{3}\right)
\end{array}\right)
$$

The kinetic terms for the vectors can then be written as

$$
\mathcal{N}_{\Lambda \Sigma}=\left(\begin{array}{ccccc}
1 & 0 & 0 & 0 & 0 \\
0 & 1 & 0 & 0 & 0 \\
0 & 0 & 1 & 0 & 0 \\
0 & 0 & 0 & \cosh \left(2 \phi_{3}\right) & -\sinh \left(2 \phi_{3}\right) \\
0 & 0 & 0 & -\sinh \left(2 \phi_{3}\right) & \cosh \left(2 \phi_{3}\right)
\end{array}\right),
$$

and the quantities in the fermionic variations read

$$
\begin{aligned}
S_{A B} & =\frac{i}{4}\left(g \cosh \left(\phi_{3}\right) e^{\sigma}+m e^{-3 \sigma}\right) \epsilon_{A B}, \\
N_{A B} & =\frac{1}{4}\left(g \cosh \left(\phi_{3}\right) e^{\sigma}-3 m e^{-3 \sigma}\right) \epsilon_{A B}, \\
M_{A B} & =-2 g \sinh \left(\phi_{3}\right) e^{\sigma} \sigma_{A B}^{3} .
\end{aligned}
$$

The other fields that are turned on are the metric, the dilaton $\sigma$ and the two-form $B_{\mu \nu}$. It is consistent to set $H_{\mu \nu \lambda}=0$ but $B_{\mu \nu}$ is not in general zero and its value can be found by solving its equations of motion [37].

We believe that after all this simplification the theory is a consistent truncation of massive type IIA supergravity on the warped background $\mathrm{AdS}_{6} \times S^{4}$. We give evidence for this in section 4 where we match the ten-dimensional result found in [43].

We finish the discussion of the matter coupled theory with an argument about the definition of the R-symmetry for all asymptotically $\mathrm{AdS}_{6}$ solutions in the theory. Let us first recall that the detailed match between supergravity and field theory for asymptotically $\mathrm{AdS}_{4}$ black holes was facilitated by the gravitational answer for the R-symmetry along the holographic renormalization group (RG) flow [7], telling us explicitly how the R-symmetry mixing is parametrized by the values of the scalar fields. In four-dimensional gauged supergravity the R-symmetry was carefully derived via the Dirac bracket of the supercharges $\mathcal{Q}$ obtained from the Noether procedure [51, 52]. Following rigorously all these steps in six dimensions is out of our scope here; however, we can still provide some solid arguments and derive the expected gravitational R-symmetry as an explicit scalar dependent combination of the two U(1)'s mixing along the flow, $F_{\mu \nu}^{r=3}$ and $F_{\mu \nu}^{I=1}=F_{\mu \nu}^{\Lambda=4}$. This proposal is strongly backed up by the agreement with the field theory results we provide in the following sections.

It is reasonable to expect that, in analogy to the four-dimensional arguments in [51, 52], the anti-commutator between two supercharges for asymptotically $\mathrm{AdS}_{6}$ solutions is given 
by a surface integral ${ }^{7}$

$$
\{\mathcal{Q}, \mathcal{Q}\} \propto \int_{\partial V} \mathrm{~d} \Sigma_{\mu \nu} \epsilon^{\mu \nu \rho \sigma \gamma \delta} \bar{\epsilon}^{A} \gamma_{\rho \sigma \gamma} \widetilde{D}_{\delta} \epsilon_{A}
$$

where $\epsilon_{A}$ is the Killing spinor preserved by $\mathrm{AdS}_{6}$, and the super-covariant derivative $\widetilde{D}$ includes all terms on the right hand side of the gravitino variation in (2.4), i.e. $\delta \psi_{A \mu}=$ $\widetilde{D}_{\mu} \epsilon_{A}$. The above anti-commutator is the explicit field dependent realization of the abstract $\mathrm{AdS}_{6}$ superalgebra, $\mathrm{F}(4)$, generating a combination of different asymptotic bosonic charges of the $S \mathrm{O}(5,2) \times \mathrm{SU}(2)_{R}$ generators. We are interested in the term in the gravitino variation in (2.4) proportional to $T_{(A B)}$, which precisely enters in the definition of the conserved $\mathrm{SU}(2)$ R-charge. We are further breaking the R-symmetry down to U(1) so we only need to look at the part proportional to $\sigma_{A B}^{3}$, cf. (2.5). We are then led to the following formula for the conserved U(1) R-symmetry charge of a given solution,

$$
R_{\mathrm{U}(1)} \propto \int_{\partial V} \mathrm{~d} \Sigma_{\mu \nu} e^{-\sigma}\left(L^{-1}\right)_{r=3 \mid \Lambda}\left(F^{\Lambda}\right)^{\mu \nu} .
$$

Note that we are only interested to know the R-symmetry at a given radial slice of the spacetime (that when interpreted as a holographic RG flow becomes a measure of how the R-symmetry changes along the flow), not at the value of the asymptotic conserved charge. Therefore, we can extract a normalized version of the integrand that we hope to match with the R-symmetry mixing in field theory. Considering that $L_{33}^{-1}=\cosh \left(\phi_{3}\right), L_{34}^{-1}=-\sinh \left(\phi_{3}\right)$ and that the democratic choice of U(1)'s corresponds to taking $F_{1, \mu \nu} \equiv F_{3, \mu \nu}+F_{4, \mu \nu}$, $F_{2, \mu \nu} \equiv F_{3, \mu \nu}-F_{4, \mu \nu}$ we finally define

$$
R_{\text {sugra }} \equiv X^{1} F_{1}+X^{2} F_{2},
$$

where the mixing of the democratic $\mathrm{U}(1)$ symmetries $F_{1,2}$ is given by the scalar dependent quantities

$$
\frac{X^{1}}{\pi} \equiv 1+\tanh \left(\phi_{3}\right), \quad \frac{X^{2}}{\pi} \equiv 1-\tanh \left(\phi_{3}\right), \quad e^{\phi_{3}}=\left(\frac{X^{1}}{X^{2}}\right)^{1 / 2} .
$$

\section{The $\mathrm{AdS}_{6}$ vacuum}

The $\mathrm{F}(4)$ supergravity discussed in the previous section has an $\mathrm{AdS}_{6}$ vacuum if we set $g=3 m[40-42]$. Indeed, considering a background with metric

$$
\mathrm{d} s^{2}=e^{2 f(r)}\left(\mathrm{d} t^{2}-\mathrm{d} r^{2}-\sum_{i=1}^{4} \mathrm{~d} x_{i}^{2}\right)
$$

\footnotetext{
${ }^{7}$ We are evaluating the Dirac bracket of two conserved asymptotic supercharges. Therefore, the resulting surface integral is defined on a space-like slice of the asymptotic AdS boundary $\partial V$ and the standard notation is

$$
\mathrm{d} \Sigma_{\mu \nu} \propto \frac{1}{\operatorname{det}\left(g_{\mu \nu}\right)} \epsilon_{\mu \nu \rho \sigma \gamma \delta} \mathrm{d} x^{\rho} \wedge \mathrm{d} x^{\sigma} \wedge \mathrm{d} x^{\gamma} \wedge \mathrm{d} x^{\delta} .
$$
}


a nontrivial scalar profile for $\sigma(r)$ and $\phi_{3}(r)$, and setting all other fields to zero, the BPS equations $(2.4)$ reduce to ${ }^{8}$

$$
\begin{aligned}
& 0=e^{-f} f^{\prime}+\frac{1}{2}\left(g \cosh \left(\phi_{3}\right) e^{\sigma}+m e^{-3 \sigma}\right), \\
& 0=e^{-f} \sigma^{\prime}-\frac{1}{2}\left(g \cosh \left(\phi_{3}\right) e^{\sigma}-3 m e^{-3 \sigma}\right), \\
& 0=e^{-f} \phi_{3}^{\prime}-2 g \sinh \left(\phi_{3}\right) e^{\sigma},
\end{aligned}
$$

where prime denotes the derivative of the function with respect to the radial coordinate $r$. With $g=3 m$, the $\mathrm{AdS}_{6}$ background corresponds to $e^{-2 f}=r^{2}$ and $\sigma=\phi_{3}=0$. We have further set $m=1 / 2$ so that the $\mathrm{AdS}_{6}$ radius is normalized to one.

A more suggestive way of solving the above equations is by taking the Ansatz $e^{2 f(r)}=$ $e^{2 f_{0}} / r^{2}$, and $\sigma, \phi_{3}$ independent of $r$. We can write the BPS equations in an alternative form by using the parameterization (2.12). The BPS equations for the fields in the gravity multiplet in terms of $X^{1,2}$ can be solved as

$$
e^{\sigma}=\frac{1}{\pi^{1 / 4}}\left(X^{1} X^{2}\right)^{1 / 8}, \quad e^{f_{0}}=e^{3 \sigma} .
$$

The on-shell supergravity action is given by

$$
\mathcal{I}_{\mathrm{AdS}_{6}}\left(X^{I}\right)=-\frac{\pi^{2} e^{4 f_{0}}}{3 G_{\mathrm{N}}}=-\frac{1}{3 \pi G_{\mathrm{N}}}\left(X^{1} X^{2}\right)^{3 / 2}
$$

We then see that the BPS equation for $\phi_{3}$, which implies $\phi_{3}=0$, is equivalent to extremizing $\mathcal{I}_{\mathrm{AdS}_{6}}$ with respect to $X^{I}$.

The function $\mathcal{I}_{\mathrm{AdS}_{6}}\left(X^{I}\right)$ has a natural field theory interpretation. The $S^{5}$ free energy of the $\operatorname{USp}(2 N)$ theory reads [53]

$$
F_{S^{5}}=-\frac{9 \sqrt{2} \pi}{5} \frac{N^{5 / 2}}{\sqrt{8-N_{f}}} .
$$

This can be generalized to the case where a mass parameter is turned on for $\mathrm{U}(1) \subset$ $\mathrm{SU}(2)_{M}[54]^{9}$

$$
F_{S^{5}}\left(\Delta_{I}\right)=-\frac{9 \sqrt{2}}{5 \pi^{2}} \frac{N^{5 / 2}}{\sqrt{8-N_{f}}}\left(\Delta_{1} \Delta_{2}\right)^{3 / 2}
$$

where $\Delta_{1}+\Delta_{2}=2 \pi$ and the extremal value is recovered for $\Delta_{1}=\Delta_{2}=\pi$. Upon using the standard $\mathrm{AdS}_{6} / \mathrm{CFT}_{5}$ dictionary [53]

$$
G_{\mathrm{N}}=\frac{5 \pi}{27 \sqrt{2}} \frac{\sqrt{8-N_{f}}}{N^{5 / 2}}
$$

\footnotetext{
${ }^{8}$ One can derive these equations by taking the ultraviolet limit of the more general flow equations (4.5), (5.6), or (6.5).

${ }^{9}$ What we denote as chemical potentials here are actually mass parameters for the antisymmetric matter field in the $S^{5}$ free energy. Comparing to [54] we have $\Delta_{1}=\pi\left(1+\frac{2 i}{3} m_{\mathrm{as}}\right), \Delta_{2}=\pi\left(1-\frac{2 i}{3} m_{\mathrm{as}}\right)$.
} 
and identifying $X^{I} \equiv \Delta_{I}$, we find that

$$
F_{S^{5}}\left(\Delta_{I}\right)=\mathcal{I}_{\mathrm{AdS}_{6}}\left(X^{I}\right)
$$

Interestingly, as shown in [32], the same quantity is also related to the Seiberg-Witten prepotential of the five-dimensional theory on $\mathbb{R}^{4} \times S^{1}$ which can be written as

$$
\mathcal{F}\left(\Delta_{I}\right)=-\frac{2 \pi i}{27} F_{S^{5}}\left(\Delta_{I}\right)=-\frac{2 \pi i}{27} \mathcal{I}_{\mathrm{AdS}_{6}}\left(X^{I}\right) .
$$

As discussed in the introduction, the function $\mathcal{I}_{\mathrm{AdS}_{6}}\left(X^{I}\right)$ in six dimensions plays a role similar to the prepotential of four-dimensional $\mathcal{N}=2$ gauged supergravity. In the $\mathrm{AdS}_{4}$ black hole story, the supergravity prepotential is similarly related both to the twisted superpotential and to the $S^{3}$ free energy of the dual field theory $[7,10,17]{ }^{10}$

\section{The $\mathrm{AdS}_{4} \times \Sigma_{\mathfrak{g}}$ solution}

The $\mathrm{F}(4)$ gauged supergravity has also an $\mathrm{AdS}_{4} \times \Sigma_{\mathfrak{g}}$ solution corresponding to the twisted compactification of the five-dimensional SCFT on a Riemann surface $\Sigma_{\mathfrak{g}}$ of genus $\mathfrak{g}$. In the infrared the field theory flows to a three-dimensional SCFT.

We consider the following Ansatz for the metric

$$
\mathrm{d} s^{2}=e^{2 f(r)}\left(\mathrm{d} t^{2}-\mathrm{d} r^{2}-\mathrm{d} z_{1}^{2}-\mathrm{d} z_{2}^{2}\right)-e^{2 h(r)} \mathrm{d} s_{\Sigma_{\mathfrak{g}}}^{2},
$$

and for the gauge fields $\mathrm{U}(1) \times \mathrm{U}(1) \subset \mathrm{SU}(2)_{R} \times \mathrm{U}(1)$ :

$$
F^{r=3}=\frac{\zeta}{g} \kappa \operatorname{vol}\left(\Sigma_{\mathfrak{g}}\right), \quad F^{I=1}=\frac{\zeta}{g} p \operatorname{vol}\left(\Sigma_{\mathfrak{g}}\right),
$$

with $\zeta= \pm 1$. There is a nontrivial profile for the scalars $\sigma(r), \phi_{3}(r)$ and all other fields are set to zero. Here, $\Sigma_{\mathfrak{g}}$ is a Riemann surface with metric normalized as $R_{\mu \nu}=\kappa g_{\mu \nu}$, with $\kappa=1$ for $S^{2}, \kappa=0$ for $T^{2}$, and $\kappa=-1$ for $\mathfrak{g}>1$. With this normalization $\operatorname{vol}\left(\Sigma_{\mathfrak{g}}\right)=2 \pi \eta_{\mathfrak{g}}$ with $\eta_{\mathfrak{g}}=2|\mathfrak{g}-1|$ for $\mathfrak{g} \neq 1$ and $\eta_{\mathfrak{g}}=1$ for $\mathfrak{g}=1$. The $\mathrm{U}(1) \subset \mathrm{SU}(2)_{R}$ gauge field is chosen in order to cancel the spin connection while the magnetic flux $p$ parameterizes a family of three-dimensional SCFTs.

If we choose spinors satisfying

$$
\gamma^{34} \epsilon_{A}=-i \zeta \sigma_{A B}^{3} \epsilon^{B}
$$

where the frame indices 3, 4 refer to the Riemann surface, the $\mathrm{U}(1) \subset \mathrm{SU}(2)_{R}$ gauge field cancels the spin connection along $\Sigma_{\mathfrak{g}}$. This is precisely the topological twist. Requiring in addition that

$$
\gamma^{\hat{r}} \epsilon_{A}=-i \epsilon_{A}
$$

\footnotetext{
${ }^{10}$ For black holes in $\mathrm{AdS}_{4} \times S^{7}$ the prepotential is proportional to the function $\sqrt{X^{1} X^{2} X^{3} X^{4}}$ with $\sum_{I=1}^{4} X^{I}=2 \pi$ and for massive type IIA black holes to $\left(X^{1} X^{2} X^{3}\right)^{2 / 3}$ with $\sum_{I=1}^{3} X^{I}=2 \pi$.
} 
where $\hat{r}$ is a frame index along the radial direction, the BPS equations (2.4) reduce to ${ }^{11}$

$$
\begin{aligned}
& 0=e^{-f} f^{\prime}-\frac{1}{8 g} e^{-\sigma-2 h}\left(\kappa \cosh \left(\phi_{3}\right)-p \sinh \left(\phi_{3}\right)\right)+\frac{1}{2}\left(g \cosh \left(\phi_{3}\right) e^{\sigma}+m e^{-3 \sigma}\right), \\
& 0=e^{-f} h^{\prime}+\frac{3}{8 g} e^{-\sigma-2 h}\left(\kappa \cosh \left(\phi_{3}\right)-p \sinh \left(\phi_{3}\right)\right)+\frac{1}{2}\left(g \cosh \left(\phi_{3}\right) e^{\sigma}+m e^{-3 \sigma}\right), \\
& 0=e^{-f} \sigma^{\prime}+\frac{1}{8 g} e^{-\sigma-2 h}\left(\kappa \cosh \left(\phi_{3}\right)-p \sinh \left(\phi_{3}\right)\right)-\frac{1}{2}\left(g \cosh \left(\phi_{3}\right) e^{\sigma}-3 m e^{-3 \sigma}\right), \\
& 0=e^{-f} \phi_{3}^{\prime}+\frac{1}{2 g} e^{-\sigma-2 h}\left(p \cosh \left(\phi_{3}\right)-\kappa \sinh \left(\phi_{3}\right)\right)-2 g \sinh \left(\phi_{3}\right) e^{\sigma} .
\end{aligned}
$$

We choose the parameterization of the scalar field $\phi_{3}$ as in (2.12) and, in addition, we introduce a redundant but democratic parameterization for the flux

$$
\mathfrak{s}_{1} \equiv 1-\mathfrak{g}+\frac{\eta_{\mathfrak{g}}}{2} p, \quad \mathfrak{s}_{2} \equiv 1-\mathfrak{g}-\frac{\eta_{\mathfrak{g}}}{2} p,
$$

with $\mathfrak{s}_{1}+\mathfrak{s}_{2}=2(1-\mathfrak{g})$. We look for $\mathrm{AdS}_{4} \times \Sigma_{\mathfrak{g}}$ vacua where $e^{f(r)}=e^{f_{0}} / r$ and $h(r), \sigma(r)$ and $\phi_{3}(r)$ are constant. Using the BPS equations (4.5), the fields in the gravity multiplet can be solved in terms of the $X^{I}$ 's as

$$
e^{\sigma}=\left(\frac{2}{3 \pi}\right)^{1 / 4}\left(X^{1} X^{2}\right)^{1 / 8}, \quad e^{h}=\frac{1}{\sqrt{3 \eta_{\mathfrak{g}}}} \frac{\left(-\mathfrak{s}_{1} X^{2}-\mathfrak{s}_{2} X^{1}\right)^{1 / 2}}{\left(X^{1} X^{2}\right)^{1 / 4}} e^{\sigma}, \quad e^{f_{0}}=e^{3 \sigma},
$$

where we set $g=3 m$ and $m=1 / 2$. The on-shell supergravity action can be written as

$$
\mathcal{I}_{\mathrm{AdS}_{4}}\left(X^{I}\right)=\frac{\pi e^{2 f_{0}+2 h} \operatorname{vol}\left(\Sigma_{\mathfrak{g}}\right)}{2 G_{\mathrm{N}}}=-\frac{4}{27 G_{\mathrm{N}}}\left(X^{1} X^{2}\right)^{1 / 2}\left(\mathfrak{s}_{2} X^{1}+\mathfrak{s}_{1} X^{2}\right) .
$$

It turns out that the BPS equation for $\phi_{3}$ - last line of (4.5) - is equivalent to the extremization of $\mathcal{I}_{\mathrm{AdS}_{4}}\left(X^{I}\right)$ with respect to $X^{I}$. The previous expression can be more elegantly rewritten as

$$
\mathcal{I}_{\mathrm{AdS}_{4}}\left(X^{I}\right)=-\frac{8}{81 G_{\mathrm{N}}} \sum_{I=1}^{2} \mathfrak{s}_{I} \frac{\partial\left(X^{1} X^{2}\right)^{3 / 2}}{\partial X^{I}}
$$

As expected, using (3.7) and identifying $X^{I} \equiv \Delta_{I}$, we find that

$$
F_{S^{3} \times \Sigma_{\mathfrak{g}}}\left(\Delta_{I}\right)=\mathcal{I}_{\mathrm{AdS}_{4}}\left(X^{I}\right)
$$

where $F_{S^{3} \times \Sigma_{\mathfrak{g}}}$ is the $S^{3} \times \Sigma_{\mathfrak{g}}$ free energy of the same theory, as a function of R-charges, computed in $[33]^{12}$

$$
F_{S^{3} \times \Sigma_{\mathfrak{g}}}\left(\Delta_{I}\right)=-\frac{8 \sqrt{2}}{15 \pi} \frac{N^{5 / 2}}{\sqrt{8-N_{f}}} \sum_{I=1}^{2} \mathfrak{s}_{I} \frac{\partial\left(\Delta_{1} \Delta_{2}\right)^{3 / 2}}{\partial \Delta_{I}}
$$

\footnotetext{
${ }^{11}$ Here we correct a numerical factor in the gaugino variation in [48].

${ }^{12}$ Comparing to [33] we have $\mathfrak{s}_{1}=(1-\mathfrak{g})\left(1+\hat{\mathfrak{n}}_{M}\right), \mathfrak{s}_{2}=(1-\mathfrak{g})\left(1-\hat{\mathfrak{n}}_{M}\right), \Delta_{1}=\pi\left(1+\widetilde{\nu}_{\mathrm{AS}}\right), \Delta_{2}=\pi\left(1-\widetilde{\nu}_{\mathrm{AS}}\right)$.
} 
Here, $\Delta_{1}+\Delta_{2}=2 \pi$. Moreover, as noticed in [32], this expression is also related to the effective twisted superpotential $\widetilde{\mathcal{W}}$ of the theory compactified on $\Sigma_{\mathfrak{g}} \times S^{1}$ :

$$
\widetilde{\mathcal{W}}\left(\Delta_{I}\right)=\frac{\pi i}{2} F_{S^{3} \times \Sigma_{\mathfrak{g}}}\left(\Delta_{I}\right)=\frac{\pi i}{2} \mathcal{I}_{\mathrm{AdS}_{4}}\left(X^{I}\right)
$$

The extremization of $F_{S^{3} \times \Sigma_{\mathfrak{g}}}\left(\Delta_{I}\right)$ with respect to $\Delta_{I}$ determines the exact R-symmetry of the three-dimensional field theory that is obtained by twisted compactification on $\Sigma_{\mathfrak{g}}$. The critical value of $F_{S^{3} \times \Sigma_{\mathfrak{g}}}\left(\Delta_{I}\right)$ is the free energy of the theory and coincides with the value derived directly in ten-dimensional massive type IIA supergravity in [43]. This is an evidence that the gauged supergravity provides a consistent truncation of the tendimensional theory.

\section{The $\mathrm{AdS}_{2} \times \Sigma_{\mathfrak{g}_{1}} \times \Sigma_{\mathfrak{g}_{2}}$ solution}

Now we search for black hole horizon solutions of the form $\operatorname{AdS}_{2} \times \Sigma_{\mathfrak{g}_{1}} \times \Sigma_{\mathfrak{g}_{2}}$. We consider the following Ansatz for the metric

$$
\mathrm{d} s^{2}=e^{2 f(r)}\left(\mathrm{d} t^{2}-\mathrm{d} r^{2}\right)-e^{2 h_{1}(r)} \mathrm{d} s_{\Sigma_{\mathfrak{g}_{1}}}^{2}-e^{2 h_{2}(r)} \mathrm{d} s_{\Sigma_{\mathfrak{g}_{2}}}^{2}
$$

and the gauge fields

$$
F^{r=3}=\frac{\zeta}{g} \kappa_{1} \operatorname{vol}\left(\Sigma_{\mathfrak{g}_{1}}\right)+\frac{\zeta}{g} \kappa_{2} \operatorname{vol}\left(\Sigma_{\mathfrak{g}_{2}}\right), \quad F^{I=1}=\frac{\zeta}{g} p_{1} \operatorname{vol}\left(\Sigma_{\mathfrak{g}_{1}}\right)+\frac{\zeta}{g} p_{2} \operatorname{vol}\left(\Sigma_{\mathfrak{g}_{2}}\right),
$$

with $\zeta= \pm 1$ and the previous conventions for Riemann surfaces. The $\mathrm{U}(1) \subset \mathrm{SU}(2)_{R}$ gauge field is chosen in order to cancel the spin connection and $p_{1}$ and $p_{2}$ are magnetic charges, one for each Riemann surface. There is as usual a nontrivial profile for the scalars $\sigma(r), \phi_{3}(r)$. This time the two-form $B_{\mu \nu}$ cannot be set to zero. Assuming $H_{\mu \nu \lambda}=0$, the equations of motion require that

$$
e^{-2 \sigma} m^{2} \mathcal{N}_{00} B^{\mu \nu}+\frac{1}{16} \epsilon^{\mu \nu \tau \rho \lambda \sigma} \eta_{\Lambda \Sigma} F_{\tau \rho}^{\Lambda} F_{\lambda \sigma}^{\Sigma}=0
$$

which is solved by

$$
B_{t r}=-\frac{\left(p_{1} p_{2}-\kappa_{1} \kappa_{2}\right)}{8 m^{2} g^{2}} e^{2 \sigma+2 f-2 h_{1}-2 h_{2}}
$$

With the spinor projections

$$
\gamma^{12} \epsilon_{A}=-i \zeta \sigma_{A B}^{3} \epsilon^{B}, \quad \gamma^{34} \epsilon_{A}=-i \zeta \sigma_{A B}^{3} \epsilon^{B}, \quad \gamma^{\hat{r}} \epsilon_{A}=-i \epsilon_{A},
$$

where the frame indices 1,2 refer to the first Riemann surface and 3,4 to the second, the $\mathrm{U}(1) \subset \mathrm{SU}(2)_{R}$ gauge field cancels the spin connection, and the BPS equations (2.4) reduce 
to

$$
\begin{aligned}
& 0=e^{-f} f^{\prime}-\frac{1}{8 g} e^{-\sigma-2 h_{1}}\left(\kappa_{1} \cosh \left(\phi_{3}\right)-p_{1} \sinh \left(\phi_{3}\right)\right) \\
& -\frac{1}{8 g} e^{-\sigma-2 h_{2}}\left(\kappa_{2} \cosh \left(\phi_{3}\right)-p_{2} \sinh \left(\phi_{3}\right)\right) \\
& +\frac{1}{2}\left(g \cosh \left(\phi_{3}\right) e^{\sigma}+m e^{-3 \sigma}\right)-\frac{3\left(p_{1} p_{2}-\kappa_{1} \kappa_{2}\right)}{32 m g^{2}} e^{\sigma-2 h_{1}-2 h_{2}}, \\
& 0=e^{-f} h_{1}^{\prime}+\frac{3}{8 g} e^{-\sigma-2 h_{1}}\left(\kappa_{1} \cosh \left(\phi_{3}\right)-p_{1} \sinh \left(\phi_{3}\right)\right) \\
& -\frac{1}{8 g} e^{-\sigma-2 h_{2}}\left(\kappa_{2} \cosh \left(\phi_{3}\right)-p_{2} \sinh \left(\phi_{3}\right)\right) \\
& +\frac{1}{2}\left(g \cosh \left(\phi_{3}\right) e^{\sigma}+m e^{-3 \sigma}\right)+\frac{\left(p_{1} p_{2}-\kappa_{1} \kappa_{2}\right)}{32 m g^{2}} e^{\sigma-2 h_{1}-2 h_{2}}, \\
& 0=e^{-f} h_{2}^{\prime}-\frac{1}{8 g} e^{-\sigma-2 h_{1}}\left(\kappa_{1} \cosh \left(\phi_{3}\right)-p_{1} \sinh \left(\phi_{3}\right)\right) \\
& +\frac{3}{8 g} e^{-\sigma-2 h_{2}}\left(\kappa_{2} \cosh \left(\phi_{3}\right)-p_{2} \sinh \left(\phi_{3}\right)\right) \\
& +\frac{1}{2}\left(g \cosh \left(\phi_{3}\right) e^{\sigma}+m e^{-3 \sigma}\right)+\frac{\left(p_{1} p_{2}-\kappa_{1} \kappa_{2}\right)}{32 m g^{2}} e^{\sigma-2 h_{1}-2 h_{2}}, \\
& 0=e^{-f} \sigma^{\prime}+\frac{1}{8 g} e^{-\sigma-2 h_{1}}\left(\kappa_{1} \cosh \left(\phi_{3}\right)-p_{1} \sinh \left(\phi_{3}\right)\right) \\
& +\frac{1}{8 g} e^{-\sigma-2 h_{2}}\left(\kappa_{2} \cosh \left(\phi_{3}\right)-p_{2} \sinh \left(\phi_{3}\right)\right) \\
& -\frac{1}{2}\left(g \cosh \left(\phi_{3}\right) e^{\sigma}-3 m e^{-3 \sigma}\right)-\frac{\left(p_{1} p_{2}-\kappa_{1} \kappa_{2}\right)}{32 m g^{2}} e^{\sigma-2 h_{1}-2 h_{2}}, \\
& 0=e^{-f} \phi_{3}^{\prime}+\frac{1}{2 g} e^{-\sigma-2 h_{1}}\left(p_{1} \cosh \left(\phi_{3}\right)-\kappa_{1} \sinh \left(\phi_{3}\right)\right) \\
& +\frac{1}{2 g} e^{-\sigma-2 h_{2}}\left(p_{2} \cosh \left(\phi_{3}\right)-\kappa_{2} \sinh \left(\phi_{3}\right)\right)-2 g \sinh \left(\phi_{3}\right) e^{\sigma} \text {. }
\end{aligned}
$$

We choose the parameterization (2.12) for the scalar field $\phi_{3}$ and a democratic parameterization for the fluxes

$$
\begin{aligned}
\mathfrak{s}_{1} \equiv 1-\mathfrak{g}_{1}+\frac{\eta_{\mathfrak{g}_{1}}}{2} p_{1}, & \mathfrak{s}_{2} \equiv 1-\mathfrak{g}_{1}-\frac{\eta_{\mathfrak{g}_{1}}}{2} p_{1}, \\
\mathfrak{t}_{1} \equiv 1-\mathfrak{g}_{2}+\frac{\eta_{\mathfrak{g}_{2}}}{2} p_{2}, & \mathfrak{t}_{2} \equiv 1-\mathfrak{g}_{2}-\frac{\eta_{\mathfrak{g}_{2}}}{2} p_{2},
\end{aligned}
$$

with $\mathfrak{s}_{1}+\mathfrak{s}_{2}=2\left(1-\mathfrak{g}_{1}\right)$ and $\mathfrak{t}_{1}+\mathfrak{t}_{2}=2\left(1-\mathfrak{g}_{2}\right)$. To have a black hole horizon $\mathrm{AdS}_{2} \times$ $\Sigma_{\mathfrak{g}_{1}} \times \Sigma_{\mathfrak{g}_{2}}$ we set $e^{f(r)}=e^{f_{0}} / r$ and $h_{1}(r), h_{2}(r), \sigma(r)$ and $\phi_{3}(r)$ constant. Using the BPS equations (5.6), the fields in the gravity multiplet can be solved in terms of $X^{I}$ as

$$
\begin{aligned}
e^{\sigma} & =\left(X^{1} X^{2}\right)^{1 / 8}\left(\frac{\left(\mathfrak{s}_{1} X^{2}+\mathfrak{s}_{2} X^{1}\right)\left(\mathfrak{t}_{1} X^{2}+\mathfrak{t}_{2} X^{1}\right)+2 X^{1} X^{2}\left(\mathfrak{s}_{2} \mathfrak{t}_{1}+\mathfrak{s}_{1} \mathfrak{t}_{2}\right)}{3 \pi\left(\mathfrak{s}_{1} X^{2}+\mathfrak{s}_{2} X^{1}\right)\left(\mathfrak{t}_{1} X^{2}+\mathfrak{t}_{2} X^{1}\right)}\right)^{1 / 4}, \\
e^{f_{0}} & =\frac{\left(X^{1} X^{2}\right)^{1 / 2}}{3 \pi} e^{-\sigma},
\end{aligned}
$$




$$
\begin{aligned}
e^{h_{1}} & =\frac{1}{\sqrt{3 \eta_{\mathfrak{g}_{1}}}} \frac{\left(-\mathfrak{s}_{1} X^{2}-\mathfrak{s}_{2} X^{1}\right)^{1 / 2}}{\left(X^{1} X^{2}\right)^{1 / 4}} e^{\sigma}, \\
e^{h_{2}} & =\frac{1}{\sqrt{3 \eta_{\mathfrak{g}_{2}}}} \frac{\left(-\mathfrak{t}_{1} X^{2}-\mathfrak{t}_{2} X^{1}\right)^{1 / 2}}{\left(X^{1} X^{2}\right)^{1 / 4}} e^{\sigma},
\end{aligned}
$$

where we set $g=3 m$ and $m=1 / 2$. One can find families of regular horizons, with fluxes satisfying all the quantization conditions, whenever $\kappa_{1}=-1$ or $\kappa_{2}=-1$. The BekensteinHawking entropy can be written as

$$
\mathcal{I}_{\mathrm{AdS}_{2}}\left(X^{I}\right)=\frac{e^{2 h_{1}+2 h_{2}} \operatorname{vol}\left(\Sigma_{\mathfrak{g}_{1}} \times \Sigma_{\mathfrak{g}_{2}}\right)}{4 G_{\mathrm{N}}}=\frac{4 \pi}{81 G_{\mathrm{N}}} \sum_{I, J=1}^{2} \mathfrak{s}_{I} \mathfrak{t}_{J} \frac{\partial^{2}\left(X^{1} X^{2}\right)^{3 / 2}}{\partial X^{I} \partial X^{J}},
$$

as a function of $X^{I}$. It is quite remarkable that the BPS equation for $\phi_{3}-$ last line of (5.6) - is equivalent to the extremization of $\mathcal{I}_{\mathrm{AdS}_{2}}\left(X^{I}\right)$ with respect to $X^{I}$. This is the attractor mechanism in six-dimensional gauged supergravity: once the fields in the gravity multiplet are expressed in terms of the scalars in the vector multiplet, the entropy is obtained by extremizing the functional $\mathcal{I}_{\mathrm{AdS}_{2}}\left(X^{I}\right)$.

We can now compare the entropy of the six-dimensional black holes with the prediction of the topologically twisted index computed in [32]. The index, at large $N$, is given by [32]

$$
\mathcal{I}_{\mathrm{SCFT}}\left(\Delta_{I}\right)=\frac{4 \sqrt{2}}{15} \frac{N^{5 / 2}}{\sqrt{8-N_{f}}} \sum_{I, J=1}^{2} \mathfrak{s}_{I} \mathfrak{t}_{J} \frac{\partial^{2}\left(\Delta_{1} \Delta_{2}\right)^{3 / 2}}{\partial \Delta_{I} \partial \Delta_{J}} .
$$

The index depends on a chemical potential $\Delta$ for the $\mathrm{U}(1)$ subgroup of the $\mathrm{SU}(2)$ global symmetry. As in [32], we find it convenient to use a pair of redundant but democratic parameters

$$
\Delta_{1}=\Delta, \quad \Delta_{2}=2 \pi-\Delta
$$

with $\Delta_{1}+\Delta_{2}=2 \pi$. In the spirit of the microscopic counting for magnetically charged AdS black holes in four dimensions, we expect that the entropy is obtained by extremizing $\mathcal{I}_{\mathrm{SCFT}}\left(\Delta_{I}\right)$ with respect to $\Delta_{I}$. This was called $\mathcal{I}$-extremization principle in $[7,8]$. Using (3.7) and identifying $X^{I} \equiv \Delta_{I}$, we find that

$$
\mathcal{I}_{\mathrm{SCFT}}\left(\Delta_{I}\right)=\mathcal{I}_{\mathrm{AdS}_{2}}\left(X^{I}\right)
$$

and we see that the field theory $\mathcal{I}$-extremization precisely corresponds to the attractor mechanism in supergravity.

\section{The $\mathrm{AdS}_{2} \times \mathcal{M}_{4}$ solution}

It is easy to find more general black hole horizons with Abelian twists. We consider the following metric

$$
\mathrm{d} s^{2}=e^{2 f(r)}\left(\mathrm{d} t^{2}-\mathrm{d} r^{2}\right)-e^{2 h(r)} \mathrm{d} s_{\mathcal{M}_{4}}^{2},
$$


where $\mathcal{M}_{4}$ is a Kähler-Einstein manifold with metric normalized as $R_{\mu \nu}=\kappa g_{\mu \nu}(\kappa= \pm 1,0)$, and gauge fields

$$
F^{r=3}=\frac{\zeta}{g} \kappa\left(e^{12}+e^{34}\right) e^{-2 h(r)}, \quad F^{I=1}=\frac{\zeta}{g} p\left(e^{12}+e^{34}\right) e^{-2 h(r)},
$$

where $e^{i}, i=1,2,3,4$, are vierbeins in the directions corresponding to the manifold $\mathcal{M}_{4}$. The reduced holonomy group on the manifold, $\mathrm{U}(2)$, splits into $\mathrm{U}(1)$ that we choose to correspond to the selfdual part of the spin connection, $\omega^{+}$, and $\mathrm{SU}(2)$ for the anti-selfdual part, $\omega^{-}$. As in the previous section, there is a nontrivial profile for the scalars $\sigma(r), \phi_{3}(r)$ and the two-form

$$
B_{t r}=-\frac{\left(p^{2}-\kappa^{2}\right)}{8 m^{2} g^{2}} e^{2 \sigma+2 f-2 h_{1}-2 h_{2}}
$$

With the spinor projections

$$
\gamma^{12} \epsilon_{A}=-i \zeta \sigma_{A B}^{3} \epsilon^{B}, \quad \gamma^{34} \epsilon_{A}=-i \zeta \sigma_{A B}^{3} \epsilon^{B}, \quad \gamma^{\hat{r}} \epsilon_{A}=-i \epsilon_{A}
$$

the $\mathrm{U}(1) \subset \mathrm{SU}(2)_{R}$ gauge field cancels the $\omega^{+}$spin connection, while the $\omega^{-}$part drops out of the Killing spinor covariant derivative, since (6.4) imply $\gamma^{1234} \epsilon_{A}=-\epsilon_{A}$. The BPS equations (2.4) reduce to

$$
\begin{aligned}
0= & e^{-f} f^{\prime}-\frac{1}{4 g} e^{-\sigma-2 h}\left(\kappa \cosh \left(\phi_{3}\right)-p \sinh \left(\phi_{3}\right)\right) \\
& +\frac{1}{2}\left(g \cosh \left(\phi_{3}\right) e^{\sigma}+m e^{-3 \sigma}\right)-\frac{3\left(p^{2}-\kappa^{2}\right)}{32 m g^{2}} e^{\sigma-4 h}, \\
0= & e^{-f} h^{\prime}+\frac{1}{4 g} e^{-\sigma-2 h}\left(\kappa \cosh \left(\phi_{3}\right)-p \sinh \left(\phi_{3}\right)\right) \\
& +\frac{1}{2}\left(g \cosh \left(\phi_{3}\right) e^{\sigma}+m e^{-3 \sigma}\right)+\frac{\left(p^{2}-\kappa^{2}\right)}{32 m g^{2}} e^{\sigma-4 h}, \\
0= & e^{-f} \sigma^{\prime}+\frac{1}{4 g} e^{-\sigma-2 h}\left(\kappa \cosh \left(\phi_{3}\right)-p \sinh \left(\phi_{3}\right)\right) \\
& -\frac{1}{2}\left(g \cosh \left(\phi_{3}\right) e^{\sigma}-3 m e^{-3 \sigma}\right)-\frac{\left(p^{2}-\kappa^{2}\right)}{32 m g^{2}} e^{\sigma-4 h}, \\
0= & e^{-f} \phi_{3}^{\prime}+\frac{1}{g} e^{-\sigma-2 h}\left(p \cosh \left(\phi_{3}\right)-\kappa \sinh \left(\phi_{3}\right)\right)-2 g \sinh \left(\phi_{3}\right) e^{\sigma} .
\end{aligned}
$$

We choose the parameterization (2.12) for the scalar field $\phi_{3}$ and a democratic parameterization for the fluxes

$$
\mathfrak{s}_{1} \equiv \kappa+p, \quad \mathfrak{s}_{2} \equiv \kappa-p,
$$

with $\mathfrak{s}_{1}+\mathfrak{s}_{2}=2 \kappa$. To have an $\mathrm{AdS}_{2} \times \mathcal{M}_{4}$ horizon topology we set $e^{f(r)}=e^{f_{0}} / r$ and $h(r), \sigma(r)$ and $\phi_{3}(r)$ constant. Using the BPS equations (6.5), the fields in the gravity multiplet can be solved in terms of $X^{I}$ as

$$
\begin{aligned}
e^{\sigma} & =\left(X_{1} X_{2}\right)^{1 / 8}\left(\frac{\left(\mathfrak{s}_{1} X^{2}+\mathfrak{s}_{2} X^{1}\right)^{2}+4 X^{1} X^{2} \mathfrak{s}_{1} \mathfrak{s}_{2}}{3 \pi\left(\mathfrak{s}_{1} X^{2}+\mathfrak{s}_{2} X^{1}\right)^{2}}\right)^{1 / 4} \\
e^{h} & =\frac{1}{\sqrt{6}} \frac{\left(-\mathfrak{s}_{1} X^{2}-\mathfrak{s}_{2} X^{1}\right)^{1 / 2}}{\left(X^{1} X^{2}\right)^{1 / 4}} e^{\sigma}, \quad e^{f_{0}}=\frac{\left(X^{1} X^{2}\right)^{1 / 2}}{3 \pi} e^{-\sigma}
\end{aligned}
$$


where we set $g=3 m$ and $m=1 / 2$. One can find regular horizons only for $\kappa=-1$ for sufficiently small $p .{ }^{13}$ The Bekenstein-Hawking entropy is then obtained by extremizing

$$
\mathcal{I}_{\mathrm{AdS}_{2}}\left(X^{I}\right)=\frac{e^{4 h} \operatorname{vol}\left(\mathcal{M}_{4}\right)}{4 G_{\mathrm{N}}}=\frac{\operatorname{vol}\left(\mathcal{M}_{4}\right)}{324 \pi G_{\mathrm{N}}} \sum_{I, J=1}^{2} \mathfrak{s}_{I} \mathfrak{s}_{J} \frac{\partial^{2}\left(X^{1} X^{2}\right)^{3 / 2}}{\partial X^{I} \partial X^{J}},
$$

with respect to $X^{I}$. Remarkably, the extremization of (6.8) is equivalent to the BPS equation for $\phi_{3}$ - last line of (6.5). This formula is very simple and suggests that the computation in [32] could be generalized to this case too. It is also reminiscent of similar expressions for $\mathrm{AdS}_{4}$ black holes (see [32] for a discussion).

\section{Acknowledgments}

We would like to thank Minwoo Suh for very useful discussions. We also like to acknowledge the collaboration with Itamar Yaakov on related topics. The work of SMH was supported by World Premier International Research Center Initiative (WPI Initiative), MEXT, Japan. KH is supported in part by the Bulgarian NSF grant DN08/3 and the bilateral grant STC/Bulgaria-France 01/6. The work of AP is supported by the Knut and Alice Wallenberg Foundation under grant Dnr KAW 2015.0083. AZ is partially supported by the INFN and ERC-STG grant 637844-HBQFTNCER. SMH would like to thank the Yau Mathematical Sciences Center of Tsinghua University in Beijing for their kind hospitality during his visit, where part of this work was done.

\section{A Conventions}

We collect here few relevant conventions and formulae used in [42]. We refer to that paper for everything missing or forgotten. Indices $A, B, \ldots$ in the fundamental representation of $\mathrm{SU}(2)_{R}$ are raised and lowered as $T^{A}=\epsilon^{A B} T_{B}$ and $T_{A}=T^{B} \epsilon_{B A}$. Indices $\Lambda, \Sigma, \ldots$ of $\mathrm{SO}\left(4, n_{\mathrm{V}}\right)$ are raised and lowered with $\eta_{\Lambda \Sigma}=\operatorname{diag}\{1,1,1,1,-1, \ldots,-1\}$. Spinors are pseudo-Majorana with $\left(\psi_{A}\right)^{\dagger} \gamma^{0}=\epsilon^{A B} \psi_{B}^{t}$ and $\gamma^{7}=i \gamma^{0} \gamma^{1} \gamma^{2} \gamma^{3} \gamma^{4} \gamma^{5} \gamma^{6}$. The potential $V$ and the fermionic shifts are constructed using the quantities

$$
A=\epsilon^{r s t} K_{r s t}, \quad B^{i}=\epsilon^{i j k} K_{j k 0}, \quad C_{I}^{t}=\epsilon^{t r s} K_{r I s}, \quad D_{I t}=K_{0 I t},
$$

where

$$
\begin{aligned}
& K_{r s \alpha}=g \epsilon_{l m n} L_{r}^{l}\left(L^{-1}\right)_{s}^{m} L_{\alpha}^{n}+g^{\prime} C_{I J K} L_{r}^{I}\left(L^{-1}\right)_{s}{ }^{J} L^{K}{ }_{\alpha}, \\
& K_{\alpha I t}=g \epsilon_{l m n} L_{\alpha}^{l}{ }_{\alpha}\left(L^{-1}\right)_{I}^{m} L^{n}{ }_{t}+g^{\prime} C_{L J K} L^{L}{ }_{\alpha}\left(L^{-1}\right)_{I}{ }^{J} L^{K}{ }_{t} .
\end{aligned}
$$

Here, $C_{I J K}$ are the structure constants of the gauge group $G \subset \mathrm{SO}\left(n_{\mathrm{V}}\right)$. For us $C_{I J K}=0$. We then have the following fermionic shifts entering the BPS equations (2.4):

$$
\begin{aligned}
S_{A B} & =\frac{i}{24}\left(A e^{\sigma}+6 m e^{-3 \sigma}\left(L^{-1}\right)_{00}\right) \epsilon_{A B}-\frac{i}{8}\left(B_{t} e^{\sigma}-2 m e^{-3 \sigma}\left(L^{-1}\right)_{i 0}\right) \gamma^{7} \sigma_{A B}^{t}, \\
N_{A B} & =\frac{1}{24}\left(A e^{\sigma}-18 m e^{-3 \sigma}\left(L^{-1}\right)_{00}\right) \epsilon_{A B}+\frac{1}{8}\left(B_{t} e^{\sigma}+6 m e^{-3 \sigma}\left(L^{-1}\right)_{i 0}\right) \gamma^{7} \sigma_{A B}^{t}, \\
M_{A B}^{I} & =\left(-C_{t}^{I}+2 i \gamma^{7} D_{t}^{I}\right) e^{\sigma} \sigma_{A B}^{t}-2 m e^{-3 \sigma}\left(L^{-1}\right)_{0}^{I} \gamma^{7} \epsilon_{A B} .
\end{aligned}
$$

\footnotetext{
${ }^{13}$ Since fluxes must be quantized, this condition puts some restriction on the choice of $\mathcal{M}_{4}$.
} 
Finally, the scalar potential reads

$$
V=-e^{2 \sigma}\left(\frac{A^{2}}{36}+\frac{B^{i} B_{i}}{4}+\frac{C^{I} C_{I t}}{4}+D^{I}{ }_{t} D_{I t}\right)+m^{2} e^{-6 \sigma} \mathcal{N}_{00}-m e^{-2 \sigma}\left(\frac{2}{3} A L_{00}-2 B^{i} L_{i 0}\right) .
$$

Open Access. This article is distributed under the terms of the Creative Commons Attribution License (CC-BY 4.0), which permits any use, distribution and reproduction in any medium, provided the original author(s) and source are credited.

\section{References}

[1] S.L. Cacciatori and D. Klemm, Supersymmetric AdS 4 black holes and attractors, JHEP 01 (2010) 085 [arXiv: 0911.4926] [INSPIRE].

[2] G. Dall'Agata and A. Gnecchi, Flow equations and attractors for black holes in $N=2 \mathrm{U}(1)$ gauged supergravity, JHEP 03 (2011) 037 [arXiv: 1012.3756] [INSPIRE].

[3] K. Hristov and S. Vandoren, Static supersymmetric black holes in $A d S_{4}$ with spherical symmetry, JHEP 04 (2011) 047 [arXiv:1012.4314] [INSPIRE].

[4] S. Katmadas, Static BPS black holes in U(1) gauged supergravity, JHEP 09 (2014) 027 [arXiv: 1405.4901] [INSPIRE].

[5] N. Halmagyi, Static BPS black holes in AdS 4 with general dyonic charges, JHEP 03 (2015) 032 [arXiv: 1408.2831] [INSPIRE].

[6] F. Benini and A. Zaffaroni, A topologically twisted index for three-dimensional supersymmetric theories, JHEP 07 (2015) 127 [arXiv: 1504.03698] [INSPIRE].

[7] F. Benini, K. Hristov and A. Zaffaroni, Black hole microstates in AdS $S_{4}$ from supersymmetric localization, JHEP 05 (2016) 054 [arXiv: 1511.04085] [INSPIRE].

[8] F. Benini, K. Hristov and A. Zaffaroni, Exact microstate counting for dyonic black holes in AdS4, Phys. Lett. B 771 (2017) 462 [arXiv: 1608.07294] [InSPIRE].

[9] F. Benini and A. Zaffaroni, Supersymmetric partition functions on Riemann surfaces, Proc. Symp. Pure Math. 96 (2017) 13 [arXiv:1605.06120] [inSPIRE].

[10] S.M. Hosseini and A. Zaffaroni, Large $N$ matrix models for $3 d \mathcal{N}=2$ theories: twisted index, free energy and black holes, JHEP 08 (2016) 064 [arXiv:1604.03122] [INSPIRE].

[11] S.M. Hosseini and N. Mekareeya, Large $N$ topologically twisted index: necklace quivers, dualities and Sasaki-Einstein spaces, JHEP 08 (2016) 089 [arXiv: 1604.03397] [INSPIRE].

[12] S.M. Hosseini, A. Nedelin and A. Zaffaroni, The Cardy limit of the topologically twisted index and black strings in $A d S_{5}, J H E P O 4$ (2017) 014 [arXiv:1611.09374] [INSPIRE].

[13] S.M. Hosseini, Black hole microstates and supersymmetric localization, Ph.D. thesis, Milan Bicocca University, Milan, Italy (2018), arXiv:1803.01863 [INSPIRE].

[14] J. Hong and J.T. Liu, The topologically twisted index of $\mathcal{N}=4$ super-Yang-Mills on $T^{2} \times S^{2}$ and the elliptic genus, JHEP 07 (2018) 018 [arXiv: 1804.04592] [INSPIRE].

[15] A. Cabo-Bizet, V.I. Giraldo-Rivera and L.A. Pando Zayas, Microstate counting of $A d S_{4}$ hyperbolic black hole entropy via the topologically twisted index, JHEP 08 (2017) 023 [arXiv: 1701.07893] [INSPIRE]. 
[16] F. Azzurli, N. Bobev, P.M. Crichigno, V.S. Min and A. Zaffaroni, A universal counting of

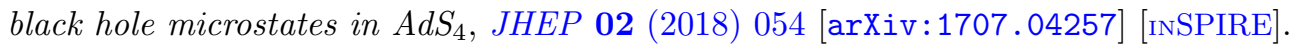

[17] S.M. Hosseini, K. Hristov and A. Passias, Holographic microstate counting for AdS $S_{4}$ black holes in massive IIA supergravity, JHEP 10 (2017) 190 [arXiv:1707.06884] [INSPIRE].

[18] F. Benini, H. Khachatryan and P. Milan, Black hole entropy in massive Type IIA, Class. Quant. Grav. 35 (2018) 035004 [arXiv:1707.06886] [INSPIRE].

[19] N. Bobev, V.S. Min and K. Pilch, Mass-deformed ABJM and black holes in AdS $S_{4}$, JHEP 03 (2018) 050 [arXiv: 1801.03135] [INSPIRE].

[20] C. Toldo and B. Willett, Partition functions on 3d circle bundles and their gravity duals, JHEP 05 (2018) 116 [arXiv:1712.08861] [INSPIRE].

[21] D. Gang and N. Kim, Large $N$ twisted partition functions in 3d-3d correspondence and Holography, arXiv: 1808.02797 [INSPIRE].

[22] J.T. Liu, L.A. Pando Zayas, V. Rathee and W. Zhao, One-loop test of quantum black holes in Anti-de Sitter space, Phys. Rev. Lett. 120 (2018) 221602 [arXiv:1711.01076] [InSPIRE].

[23] J.T. Liu, L.A. Pando Zayas, V. Rathee and W. Zhao, Toward microstate counting beyond large $N$ in localization and the dual one-loop quantum supergravity, JHEP 01 (2018) 026 [arXiv: 1707.04197] [INSPIRE].

[24] I. Jeon and S. Lal, Logarithmic corrections to entropy of magnetically charged AdS $S_{4}$ black holes, Phys. Lett. B 774 (2017) 41 [arXiv:1707. 04208] [INSPIRE].

[25] N. Halmagyi and S. Lal, On the on-shell: the action of AdS $S_{4}$ black holes, JHEP 03 (2018) 146 [arXiv: 1710.09580] [INSPIRE].

[26] A. Cabo-Bizet et al., Entropy functional and the holographic attractor mechanism, JHEP 05 (2018) 155 [arXiv: 1712.01849] [INSPIRE].

[27] K. Hristov, I. Lodato and V. Reys, On the quantum entropy function in $4 d$ gauged supergravity, JHEP 07 (2018) 072 [arXiv: 1803.05920] [INSPIRE].

[28] J. Nian and X. Zhang, Entanglement entropy of ABJM theory and entropy of topological black hole, JHEP 07 (2017) 096 [arXiv:1705. 01896] [INSPIRE].

[29] S.M. Hosseini, K. Hristov and A. Zaffaroni, An extremization principle for the entropy of rotating BPS black holes in AdS $S_{5}, J H E P 07$ (2017) 106 [arXiv:1705.05383] [INSPIRE].

[30] S.M. Hosseini, K. Hristov and A. Zaffaroni, A note on the entropy of rotating BPS $A d S_{7} \times S^{4}$ black holes, JHEP 05 (2018) 121 [arXiv:1803.07568] [INSPIRE].

[31] J.T. Liu, L.A. Pando Zayas and S. Zhou, Subleading microstate counting in the dual to massive type IIA, arXiv: 1808.10445 [INSPIRE].

[32] S.M. Hosseini, I. Yaakov and A. Zaffaroni, Topologically twisted indices in five dimensions and holography, JHEP 11 (2018) 119 [arXiv:1808.06626] [INSPIRE].

[33] P.M. Crichigno, D. Jain and B. Willett, 5D partition functions with a twist, JHEP 11 (2018) 058 [arXiv: 1808.06744] [INSPIRE].

[34] N. Seiberg, Five-dimensional SUSY field theories, nontrivial fixed points and string dynamics, Phys. Lett. B 388 (1996) 753 [hep-th/9608111] [inSPIRE].

[35] F. Benini, N. Bobev and P.M. Crichigno, Two-dimensional SCFTs from D3-branes, JHEP 07 (2016) 020 [arXiv: 1511.09462] [INSPIRE]. 
[36] N. Bobev and P.M. Crichigno, Universal RG flows across dimensions and holography, JHEP 12 (2017) 065 [arXiv: 1708.05052] [INSPIRE].

[37] M. Suh, D4-branes wrapped on supersymmetric four-cycles, arXiv:1809.03517 [INSPIRE].

[38] A. Brandhuber and Y. Oz, The D4-D8 brane system and five-dimensional fixed points, Phys. Lett. B 460 (1999) 307 [hep-th/9905148] [INSPIRE].

[39] S. Ferrara, A. Kehagias, H. Partouche and A. Zaffaroni, AdS $S_{6}$ interpretation of $5 D$ superconformal field theories, Phys. Lett. B 431 (1998) 57 [hep-th/9804006] [INSPIRE].

[40] L.J. Romans, The $F_{4}$ gauged supergravity in six-dimensions, Nucl. Phys. B 269 (1986) 691 [INSPIRE].

[41] R. D'Auria, S. Ferrara and S. Vaula, $F_{4}$ supergravity and $5 D$ superconformal field theories, Class. Quant. Grav. 18 (2001) 3181 [hep-th/0008209] [INSPIRE].

[42] L. Andrianopoli, R. D'Auria and S. Vaula, Matter coupled $F_{4}$ gauged supergravity Lagrangian, JHEP 05 (2001) 065 [hep-th/0104155] [INSPIRE].

[43] I. Bah, A. Passias and P. Weck, Holographic duals of five-dimensional SCFTs on a Riemann surface, arXiv:1807.06031 [INSPIRE].

[44] S. Ferrara, A. Marrani, J.F. Morales and H. Samtleben, Intersecting Attractors, Phys. Rev. D 79 (2009) 065031 [arXiv:0812.0050] [INSPIRE].

[45] H. Het Lam and S. Vandoren, BPS solutions of six-dimensional $(1,0)$ supergravity coupled to tensor multiplets, JHEP 06 (2018) 021 [arXiv:1804.04681] [INSPIRE].

[46] M. Suh, D4-branes wrapped on supersymmetric four-cycles from matter coupled $F(4)$ gauged supergravity, arXiv:1810.00675 [INSPIRE].

[47] M. Cvetič, H. Lü and C.N. Pope, Gauged six-dimensional supergravity from massive type IIA, Phys. Rev. Lett. 83 (1999) 5226 [hep-th/9906221] [INSPIRE].

[48] P. Karndumri, Twisted compactification of $N=25 D$ SCFTs to three and two dimensions from $F(4)$ gauged supergravity, JHEP 09 (2015) 034 [arXiv:1507.01515] [INSPIRE].

[49] M. Gutperle, J. Kaidi and H. Raj, Janus solutions in six-dimensional gauged supergravity, JHEP 12 (2017) 018 [arXiv: 1709.09204] [INSPIRE].

[50] M. Gutperle, J. Kaidi and H. Raj, Mass deformations of 5d SCFTs via holography, JHEP 02 (2018) 165 [arXiv: 1801.00730] [INSPIRE].

[51] K. Hristov, C. Toldo and S. Vandoren, On BPS bounds in $D=4 N=2$ gauged supergravity, JHEP 12 (2011) 014 [arXiv:1110.2688] [INSPIRE].

[52] K. Hristov, On BPS bounds in $D=4 N=2$ gauged supergravity II: general matter couplings and black hole masses, JHEP 03 (2012) 095 [arXiv: 1112.4289] [INSPIRE].

[53] D.L. Jafferis and S.S. Pufu, Exact results for five-dimensional superconformal field theories with gravity duals, JHEP 05 (2014) 032 [arXiv: 1207.4359] [INSPIRE].

[54] C.-M. Chang, M. Fluder, Y.-H. Lin and Y. Wang, Romans supergravity from five-dimensional holograms, JHEP 05 (2018) 039 [arXiv:1712.10313] [INSPIRE]. 